\title{
Microsatellite Instability and Life Style Factors in Sporadic Colorectal Cancer
}

\author{
Nora Atef ${ }^{1 *}$, Nelly Alieldin ${ }^{1}$, Ghada Sherif ${ }^{1}$, Iman $_{\text {Loay }}{ }^{2}$, Ahmed Mostafa \\ Mahmoud $^{3}$, Ghada Mohamed ${ }^{2}$
}

\begin{abstract}
Background: Colorectal cancer (CRC) in Egypt is a relatively high young onset disease. As a form of heterogeneous cancer, there is interplay between genetic and environmental factors. We aimed at probing the association of life style factors and Microsatellite Instability (MSI) status that could provide more insights on carcinogenic process of CRC. Methods: One hundred incident sporadic CRC patients were involved. Information on risk factors of CRC was obtained and microsatellite instability status was predicted through evaluation of MMR protein expression via immunohistochemistry (IHC). Results: Median age was 47.50 years, females represented 54.0\% and 36\% of patients were Microsatellite Instability High (MSI-H). Most patients with right sided colon cancer (78.3\%) were MSI-H while mostly stable or low MSS/MSI-L for left-sided colon and rectum $(78.6 \%, 74.3 \%$ respectively, $\mathrm{p}<0.001)$. Patients with low physical activity had higher risk of MSS/MSI-L than those with moderate or high activity $\mathrm{p}=0.026$. Patients with BMI greater than $30 \mathrm{Kg} / \mathrm{m}^{2}$ had higher MSS/MSI-L (75.5\%) than those with BMI between $25-30 \mathrm{Kg} / \mathrm{m}^{2}(60.6 \%)$ and those with normal BMI $<25(38.9 \%)$, $\mathrm{p}$ for trend $=0.006$. On subgroup analyses, the association of high BMI with MSS/ MSI-L was only shown in patients younger than 40 years, females, stage III, non-mucin secreting adenocarcinoma and a significant interaction with physical activity. Conclusion: In Conclusion, the present study confirms the increased risk of MSS/MSI-L with increased BMI and speculates this association to be modified by patient's life style and tumor characteristics. Further research is needed to validate present results.
\end{abstract}

Keywords: MSI- CRC- BMI- physical activity- Egypt

Asian Pac J Cancer Prev, 21 (5), 1471-1480

\section{Introduction}

Colorectal cancer (CRC) is one of the leading causes of mortality and morbidity in the world (Favoriti et al., 2016). In Egypt, CRC ranked the 6th, representing about $4 \%$ of total cancers in both sexes (Zeeneldin et al., 2012). $\mathrm{CRC}$ is a heterogeneous disease that can be classified into distinct subtypes with different underlying molecular alterations, such as microsatellite instability (MSI), chromosomal instability, and the CpG-island methylator phenotype (CIMP) (Jass, 2007). MSI is a kind of genomic instability where mutations occur in nucleotide repeat sequences throughout the genome. These repeat sequences are known as microsatellites, and the discrepancy between these sequences in tumor and germline cells are known as microsatellite instability (Boland and Goel, 2010). MSI arises from defects in DNA mismatch repair (MMR) system (Vilar and Gruber, 2010).

Many risk factors are known in CRC of which two modifiable and interrelated risk factors, excess body weight and physical inactivity, are reported to account for about a fourth to a third of colorectal cancers (Lee et al., 2007).

High body mass index (BMI) is an established risk factor for colorectal cancer (WCRF, 2007; Renehan et al., 2008; Ning et al., 2010; Ning et al., 2013); however, associations are usually stronger for men than women and for colon than rectal cancers (Campbell et al., 2007; Renehan etal., 2008; Ning etal., 2010; Ning et al., 2013). Emerging data suggestthat the BMI-colorectal cancer association differs by microsatellite instability (MSI) status (Slattery et al., 2000; Satia et al., 2005; Campbell etal., 2010; Hughes et al., 2012; Sinicrope et al., 2012), with stronger associations typically shown for MS-stable than MSI-high tumors.

When BMI was evaluated in the peri or post-diagnosis period, generally null or only modest associations were shown (Sinicrope et al., 2012; Campbell et al., 2012). In contrast, when pre-diagnosis BMI was evaluated, studies typically showed higher risks of all-cause and colorectal-cancer-specific mortality with high BMI (Prizment et al., 2010; Campbell et al., 2012; Parkin et

${ }^{1}$ Biostatistics and Cancer Epidemiology department, National Cancer Institute (NCI), Cairo University, Egypt. ${ }^{2}$ Pathology Department, NCI, Cairo University, Egypt. ${ }^{3}$ Surgical Oncology department , NCI, Cairo University, Egypt. *For Correspondence: noraatef148@yahoo.com 


\section{al., 2014, Fedirko et al., 2014).}

Many studies have investigated associations of risk factors and colorectal cancer by anatomic subsite of the tumor (e.g., colon/rectum, proximal/distal) (Botteri et al., 2008; Harriss et al., 2009; Ning etal., 2010), which may partly reflect associations with different molecular types of carcinogenesis (Renehan et al., 2008). Discrimination of CRC by molecular subtypes could provide clearer distinction of colorectal cancers and their associations with risk factors than tumor subsite alone (Iacopetta, 2002; Yamauchi et al., 2012). Knowledge and understanding of associations of risk factors and MSI subtypes of CRC could contribute to better understanding of the interaction of risk factors and carcinogenic processes.

The aim of this study was to show frequency of MSI in Egyptian CRC patients and whether there is an association between MSI and risk factors of CRC and if present could it be modified by BMI.

\section{Materials and Methods}

\section{Study design and study population}

Current study is cross sectional in design. Incident sporadic CRC diagnosed at NCI, Cairo University in the period from January 2017 to January 2018 were included.

Criteria of inclusion was all ages, both gender, newly diagnosed, sporadic, histologically proved primary colorectal cancer, mental ability to complete and willing to participate. Prevalent cases, those with hereditary colorectal cancer syndromes (FAP, Crohn's disease and HNPCC) who fulfill Revised Bethesda Guidelines, or advanced stage (stage IV) were not included. Out of 101 patients, one hundred agreed to participate (99\%). Sporadic colorectal cancer is the most common type of CRC and includes approximately $75 \%$ of cases that display no apparent evidence of having inheritance of disorder. Sporadic colorectal cancer is common among elder people, probably because of environmental factors, dietary, and aging (Arvelo et al., 2015).

\section{Data collection}

Personal interviews were conducted during hospitalization, before or just after surgery. Standardized questionnaire with detailed medical and family history, as well as socio-demographic and lifestyle factors was used. The index date was the date of diagnosis. Information on weight before diagnosis and current height were obtained from self-reports during the interview, to calculate BMI of the patients $\left(\mathrm{kg} / \mathrm{m}^{2}\right)$. Self-reported weight was shown to be highly accurate compared to standardized measurements (Rimm et al.,1990), weight at least 2 years before the index year was used (e.g., for a person age 65 years we used weight at age 63). This measure of body weight was preferred over current weight because cancer-related symptoms before and after diagnosis or therapy of colorectal cancer can cause weight changes. Detailed data about physical activity at work, leisure time and walking were collected during the interview by the short form of International Physical Activity Questionnaire (IPAQ) (Booth et al., 2000).

\section{Collection and immunostaining of tumor samples}

Immunohistochemistry (IHC) is now considered a validated method in predicting MSI in colorectal cancer with high sensitivity (93\%) and nearly perfect specificity in predicting MSI in CRC as determined by PCR (Lindor et al., 2002; Shia, 2015), but less certain in other cancer types. For seleting CRC cases, diagnosis was confirmed based on examining the Hematoxylin and Eosin (HE) stained sections under the light microscopy. For IHC staining, 4 sections were cut for each CRC case at a thickness of $4 \mu \mathrm{m}$ from the paraffin-embedded tissues. Sections were then placed onto X-tra adhesive slides. IHC staining was performed using BenchMark XT (Ventana) autostainer according to the protocol instruction. Birefly, deparaffinization followed by cell conditioning for 64 minutes, then antigen retrieval using reaction buffer $(\mathrm{PH}$ 7.4-7.8) was carried out. Next, ready-to-use primary monoclonal antibodies were applied under specific incubation temperature and time.

For assessment of mismatch repair (MMR) proteins (MLH1, PMS2, MSI-H2, and MSI-H6), primary antibodies used as follows: VENTANA anti-MLH1 (M1) Mouse Monoclonal Primary Antibody, cat no. 790-5091, VENTANA anti-PMS2 (A16-4) Mouse , cat no. 790-5094, VENTANA anti-MSI-H2 (G219-1129) Mouse Monoclonal Primary Antibody, cat no. 790-5093, VENTANA anti-MSI-H6 (SP93) Rabbit Monoclonal Primary Antibody, cat no. 790-5092.

The reaction is visualized by using $D A B$ (Diaminobenzidine) chromogen as a coloring agent andHematoxylin as a counterstain. Normal colonic mucosa was used as positive control.

\section{Microsatellite instability analysis}

Cases were categorized into positive (unequivocal positive nuclear staining within tumor cells) and negative (complete absence of nuclear staining within tumor cells with concurrent internal positive controls) (Hughes et al., 2012). Normal colonic mucosa, stromal fibroblasts, lymphocytes or germinal centers of lymphoid follicles were used as positive internal control) (Figures 1 and 2). Tumor displaying loss of at least one mismatch repair protein (MMR protein) can be collectively referred to as MMR-deficient (dMMR) and are considered to be (MSI-H), whereas those with intact MMR proteins can be classified as MMR-proficient (pMMR). pMMR are expected to be microsatellite stable (MSS) or MSI-low (MSI-L) (Kawakami et al., 2015).

\section{Sample size}

Based on a previous study by Soliman et al., (2001) who found that, frequency of MSI-H tumors in the Egyptian group was $37.0 \%$, while Vilar and Gruber, 2010 reported that prevalence of MSI-H ranged from $15-20 \%$. We assumed a frequency of MSI-H to be $30 \%$ and calculated the required sample size to be $81 \mathrm{CRC}$ patients which was sufficient for estimating the expected proportion with $10 \%$ absolute precision $(0.25-0.35)$ with $95 \%$ confidence interval. We increased the number to 100 to allow for non-compliance of patients. Using the above criteria, $36 \%$ showed negative expression of at least one 
of the MMR proteins and were predicted to be MSI-H (Figure 2), while 64\% showed retained expression of all MMR proteins and predicted to be MSS/MSI-L (Figure 1).

\section{Statistical analysis}

Data was analyzed using IBM SPSS advanced statistics (Statistical Package for Social Sciences), version 24 (SPSS Inc., Chicago, IL). Numerical data was described as median and range, while qualitative data was described as number and percentage. Man Whitney test used to compare non-normally distributed numerical variables. Chi-square or Fisher exact tests as appropriate were used for comparing categorical data and calculating risk. Interaction was tested using Breslow Day test. All tests were two tailed and $\mathrm{p}$ value set significant at 0.05 .

\section{Results}

Relation of MSI to patient characteristics and life-style

Out of 100 incident CRC patients, 36\% showed loss of the expression of at least one MMR protein (dMMR/ MSI-H) and 64\% showed preserved expression of all MMR proteins [pMMR/MSS and MSI-L], Figures 1 and 2. The median age was 47.50 with a range of (19-86) years and females represented $54.0 \%$. Patients aged more than 40 years were found to have slightly higher percent of MSS/MSI-L than those $\leq 40$ years and female patients compared to males without a statistical significance. School education measured in years, family history of $\mathrm{CRC}$, being hypertensive or diabetic did not show a significant relation with MSI status. Most Patients with right sided colon cancer $(78.3 \%)$ were MSI-H while it was nearly the reverse for left-sided colon and rectum (78.6\% and $74.3 \% \mathrm{MSS} / \mathrm{MSI}-\mathrm{L}$ respectively), $\mathrm{p}$ value $<0.001$. Whether the tumor was mucin or non-mucin secreting adenocarcinoma, it does not affect MSI status. No significant relation was found between tumor grade, lymph node infiltration or TNM stage and MSI status (Table1).

Former smokers tend to have a higher percent of MSS/MSI-L than current smokers without significance. History of alcohol consumption or use of oral contraceptives pills in females were not found to be related to MSI. Neither consumption of diet with high fiber content (vegetable and fruits) nor fast food diet or processed meat was found to be associated with MSI status. Patients with low physical activity (measured as $<600$ MET minutes /week) had higher percentage of MSS/ MSI-L than those with moderate or high physical activity ( $\mathrm{p}$ value $=0.026$ ). Patients with pre-diagnosis BMI greater

Table 1. Relation of MSI to Clinicopathological Characteristics of 100 CRC Patients

\begin{tabular}{|c|c|c|c|c|c|c|c|}
\hline \multirow[t]{3}{*}{ Variables } & & \multirow[b]{3}{*}{ Total $=100$} & \multicolumn{4}{|c|}{ MSI } & \multirow[t]{3}{*}{$P$-value } \\
\hline & & & \multicolumn{2}{|c|}{ MSS/MSI-L } & \multicolumn{2}{|c|}{ MSI-H } & \\
\hline & & & $\mathrm{N}=64$ & $\%$ & $\mathrm{~N}=36$ & $\%$ & \\
\hline \multirow[t]{2}{*}{ Age } & $<=40$ & 28 & 16 & 57.1 & 12 & 42.9 & 0.373 \\
\hline & $>40$ & 72 & 48 & 66.7 & 24 & 33.3 & \\
\hline \multirow[t]{2}{*}{ Sex } & Male & 44 & 27 & 61.4 & 17 & 38.6 & 0.626 \\
\hline & Female & 56 & 37 & 66.1 & 19 & 33.9 & \\
\hline \multirow[t]{4}{*}{ School Education/years } & Illiterate & 32 & 21 & 65.6 & 11 & 34.4 & 0.492 \\
\hline & $<=9$ & 24 & 14 & 58.3 & 10 & 41.7 & \\
\hline & $10-12$ & 32 & 19 & 59.4 & 13 & 40.6 & \\
\hline & $>=13$ & 12 & 10 & 83.3 & 2 & 16.7 & \\
\hline \multirow[t]{2}{*}{ HPN } & No & 80 & 51 & 63.8 & 29 & 36.3 & 0.917 \\
\hline & Yes & 20 & 13 & 65.0 & 7 & 35.0 & \\
\hline \multirow[t]{2}{*}{$\mathrm{DM}$} & No & 86 & 57 & 66.3 & 29 & 33.7 & 0.368 \\
\hline & Yes & 14 & 7 & 50.0 & 7 & 50.0 & \\
\hline \multirow[t]{3}{*}{ Tumor site } & Rt sided colon & 23 & 5 & 21.7 & 18 & 78.3 & $<0.001$ \\
\hline & Left sided colon & 42 & 33 & 78.6 & 9 & 21.4 & \\
\hline & Rectum & 35 & 26 & 74.3 & 9 & 25.7 & \\
\hline Tumor pathology & Non mucin Secreting Adenocarcinoma & 76 & 51 & 67.1 & 25 & 32.9 & 0.33 \\
\hline Tumor & Mucin Secreting Adenocarcinoma & 24 & 13 & 54.2 & 11 & 45.8 & \\
\hline \multirow[t]{2}{*}{ Grade $(\mathrm{n}=91)$} & Low & 81 & 52 & 64.2 & 29 & 35.8 & 0.492 \\
\hline & High & 10 & 5 & 50.0 & 5 & 50.0 & \\
\hline \multirow{2}{*}{$\begin{array}{l}\text { Lymph node infiltration } \\
(\mathrm{n}=98)\end{array}$} & No & 58 & 38 & 65.5 & 20 & 34.5 & 0.831 \\
\hline & Yes & 40 & 25 & 62.5 & 15 & 37.5 & \\
\hline \multirow{3}{*}{$\begin{array}{l}\text { TNM staging } \\
(\mathrm{n}=98)\end{array}$} & I & 10 & 6 & 60.0 & 4 & 40.0 & 0.866 \\
\hline & II & 48 & 32 & 66.7 & 16 & 33.3 & \\
\hline & III & 40 & 25 & 62.5 & 15 & 37.5 & \\
\hline
\end{tabular}

MSI, Microsatellite instability; MSS/MSI-L, Microsatellite stable / Microsatellite instability low; MSI-H, Microsatellite instability High; HPN, Hypertension; DM, Diabetes Mellitus 
Table 2. Relation of MSI to Life Style in 100 CRC Patients

\begin{tabular}{|c|c|c|c|c|c|c|c|}
\hline \multirow[t]{3}{*}{ Variables } & & \multirow[b]{3}{*}{ Total $=100$} & \multicolumn{4}{|c|}{ MSI } & \multirow[t]{3}{*}{$P$-value } \\
\hline & & & \multicolumn{2}{|c|}{ MSS/MSI-L } & \multicolumn{2}{|c|}{ MSI-H } & \\
\hline & & & $\mathrm{N}$ & $\%$ & $\mathrm{~N}$ & $\%$ & \\
\hline \multirow[t]{2}{*}{ Smoking status } & Never smoker & 71 & 45 & 63.4 & 26 & 36.6 & 0.84 \\
\hline & Ever Smoker (current \& former) & 29 & 19 & 65.5 & 10 & 34.5 & \\
\hline \multirow{2}{*}{$\begin{array}{l}\text { History of alcohol } \\
\text { consumption }\end{array}$} & No & 97 & 63 & 64.9 & 34 & 35.1 & 0.294 \\
\hline & Yes & 3 & 1 & 33.3 & 2 & 66.7 & \\
\hline \multirow[t]{2}{*}{ Contraceptive pills $(\mathrm{n}=56)$} & No & 36 & 22 & 61.1 & 14 & 38.9 & 0.571 \\
\hline & Yes & 20 & 14 & 70.0 & 6 & 30.0 & \\
\hline \multicolumn{2}{|c|}{ Median consumption of fruits and vegetables (Serving/day) } & 100 & \multicolumn{2}{|c|}{$0.71(0.07-2.50)$} & \multicolumn{2}{|c|}{$0.64(0.29-2.43)$} & 0.837 \\
\hline \multirow[t]{2}{*}{ Eating fast food (Habitual) } & No & 75 & 51 & 68.0 & 24 & 32.0 & 0.229 \\
\hline & Yes & 25 & 13 & 52.0 & 12 & 48.0 & \\
\hline \multirow[t]{2}{*}{ Eating processed meat } & No & 59 & 37 & 62.7 & 22 & 37.3 & 0.833 \\
\hline & Yes & 41 & 27 & 65.9 & 14 & 34.1 & \\
\hline \multirow[t]{2}{*}{ Physical activity } & Low & 59 & 43 & 72.9 & 16 & 27.1 & 0.026 \\
\hline & Moderate or high & 41 & 21 & 51.2 & 20 & 48.8 & \\
\hline \multirow[t]{3}{*}{ BMI } & $<=25$ & 18 & 7 & 38.9 & 11 & 61.1 & 0.019 \\
\hline & $25-30$ & 33 & 20 & 60.6 & 13 & 39.4 & \\
\hline & $>30$ & 49 & 37 & 75.5 & 12 & 24.5 & \\
\hline${ }^{*} P$-value for trend & & & & & & & 0.006 \\
\hline
\end{tabular}

MSI, Microsatellite instability; MSS/MSI-L, Microsatellite stable / Microsatellite instability low; MSI-H, Microsatellite instability High; BMI, Body mass index

than $30 \mathrm{Kg} / \mathrm{m}^{2}$ had higher MSS/MSI-L (75.5\%) than those with BMI between 25-30 (60.6\%) and those with normal $\mathrm{BMI}<25$ (38.9\%), p value for trend was 0.006 (Table 2).

Association of MSI, BMI and interaction with patient characteristics

After categorizing patients into MSS/MSI-L and
MSI-H, they were further classified according to BMI into; $\mathrm{BMI}<25 \mathrm{Kg} / \mathrm{m}^{2}$ and $\mathrm{BMI} \geq 25 \mathrm{Kg} / \mathrm{m}^{2}$. For all patients those with $\mathrm{BMI} \geq 25$ had more than triple increased risk of MSS/MSI-L than those with normal weight (OR 3.58 (1.24-10.31); $\mathrm{p}=0.014)$. This significant relationship was tested for effect modification by different patient characteristics. For age, the significant association of
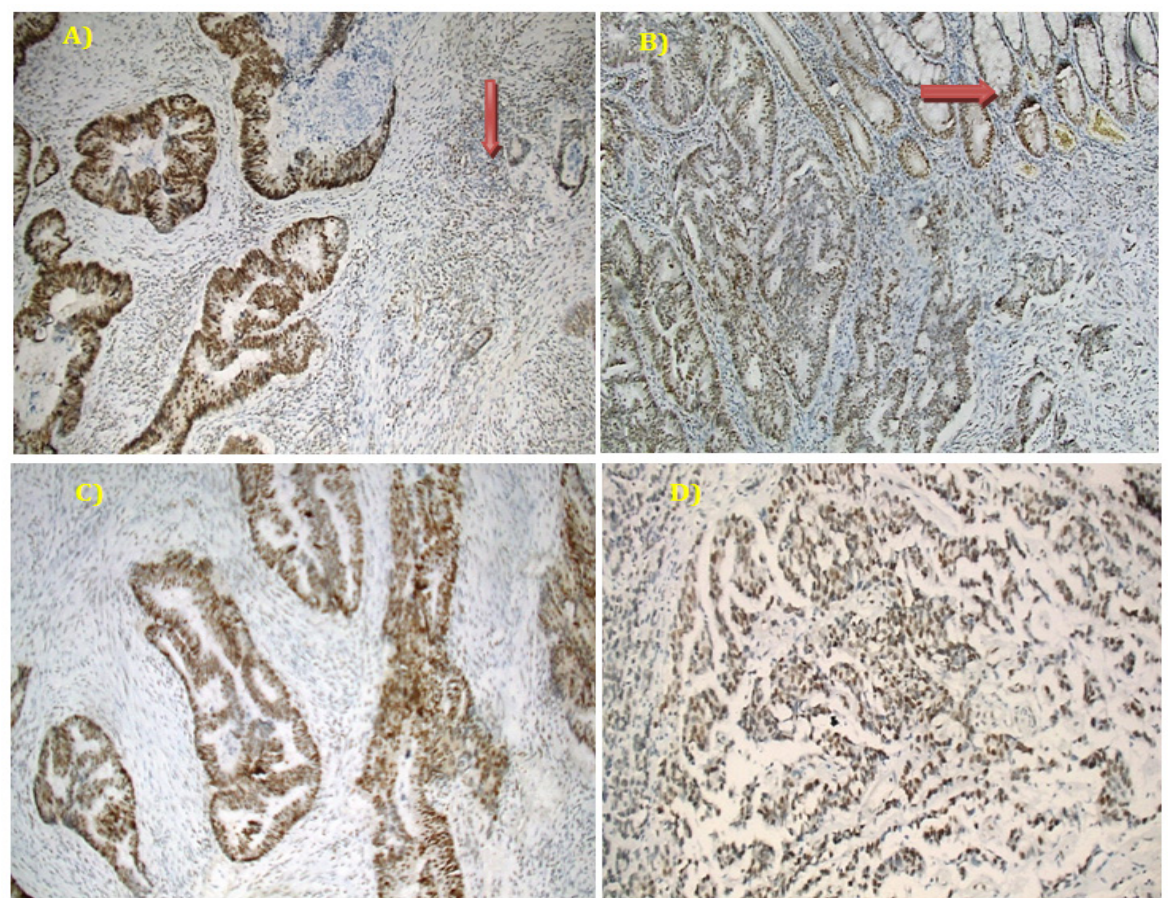

Figure 1. A Representative Example of pMMR (MSS) Colorectal Carcinoma, shows (A) Positive MLH-1 in tumor cells as well as the internal positive control [lymphocytes with red arrow] (Mag. 100X). (B) PMS2 is positive in tumor cells as well as normal colonic mucosa [red arrow] (Mag. 100X). (C) Positive MSH6 in tumor cells (Mag. 100X). (D) Positive MSH2 in tumor cells (Mag. 100X). 
DOI:10.31557/APJCP.2020.21.5.1471

Microsatellite Instability and Life Style Factors in Sporadic Colorectal Cancer

Table 3. Association of BMI with MSS/MSI-L and MSI-H Colorectal Cancer and Interaction with Patient Characteristics

\begin{tabular}{|c|c|c|c|c|c|c|}
\hline & \multicolumn{2}{|c|}{ MSS/MSI-L N=64 } & \multicolumn{2}{|c|}{ MSI-H N=36 } & \multirow[t]{4}{*}{ OR $(95 \% \mathrm{CI})^{*}$} & \multirow{4}{*}{$\begin{array}{c}P \text {-value } \\
\text { within } \\
\text { subgroup }\end{array}$} \\
\hline & $\mathrm{BMI}<25$ & $\mathrm{BMI} \geq 25$ & $\mathrm{BMI}<25$ & $\mathrm{BMI} \geq 25$ & & \\
\hline & $\mathrm{N}=7$ & $\mathrm{~N}=57$ & $\mathrm{~N}=11$ & $\mathrm{~N}=25$ & & \\
\hline & $\mathrm{N}(\%)$ & $\mathrm{N}(\%)$ & $\mathrm{N}(\%)$ & $\mathrm{N}(\%)$ & & \\
\hline \multicolumn{7}{|l|}{ Age } \\
\hline Age $<40$ & $1(14.3)$ & $15(71.4)$ & $6(85.7)$ & $6(28.6)$ & $14.93(1.47-142.9)$ & 0.023 \\
\hline Age $\geq 40$ & $6(54.5)$ & $42(68.9)$ & $5(45.5)$ & $19(31.1)$ & $1.84(0.49-6.80)$ & 0.488 \\
\hline$P$-value for interaction & & & & 0.1 & & \\
\hline \multicolumn{7}{|l|}{ Sex } \\
\hline Males & $5(50.0)$ & $22(64.7)$ & $5(50.0)$ & $12(35.3)$ & $1.83(0.44-7.63)$ & 0.401 \\
\hline Females & $2(25.0)$ & $35(72.9)$ & $6(75.0)$ & $13(27.1)$ & $8.06(1.44-45.5)$ & 0.014 \\
\hline$P$-value for interaction & & & & 0.187 & & \\
\hline \multicolumn{7}{|l|}{ Smoking } \\
\hline Never smoking & $2(20.0)$ & $43(70.5)$ & $8(80.0)$ & $18(29.0)$ & $9.5(1.85-50.0)$ & 0.004 \\
\hline Ever smoking & $5(62.5)$ & $14(66.7)$ & $3(37.5)$ & $7(33.3)$ & $1.20(0.22-6.54)$ & 1 \\
\hline$P$-value for interaction & & & & 0.078 & & \\
\hline \multicolumn{7}{|l|}{ Stage } \\
\hline Stage I\&II & $6(50.0)$ & $32(69.6)$ & $6(50.0)$ & $14(30.4)$ & $2.28(0.63-8.33)$ & 0.307 \\
\hline Stage III & $1(16.7)$ & $24(70.6)$ & $5(83.3)$ & $10(29.4)$ & $12.0(1.24-111.1)$ & 0.021 \\
\hline$P$-value for interaction & & & & 0.198 & & \\
\hline \multicolumn{7}{|l|}{ Pathology } \\
\hline Non mucin secreting adenocarcinoma & $4(33.3)$ & $47(73.4)$ & $8(66.7)$ & $17(26.6)$ & $5.52(1.47-20.83)$ & 0.007 \\
\hline Mucin and signet ring adenocarcinoma & $3(50.0)$ & $10(55.6)$ & $3(50.0)$ & $8(44.4)$ & $1.25(0.196-7.93)$ & 0.813 \\
\hline$P$-value for interaction & & & & 0.194 & & \\
\hline \multicolumn{7}{|l|}{ Site } \\
\hline Rt side & $0(0)$ & $5(27.8)$ & $5(100.0)$ & $13(72.2)$ & 1 & \\
\hline Lt side & $3(50)$ & $30(83.3)$ & $3(50)$ & $6(16.7)$ & $5.0(0.80-31.25)$ & 0.101 \\
\hline Rectum & $4(57.1)$ & $22(78.6)$ & $3(42.9)$ & $6(21.4)$ & $2.75(0.48-15.87)$ & 0.34 \\
\hline$P$-value for interaction & & & & 0.685 & & \\
\hline \multicolumn{7}{|l|}{ Physical activity } \\
\hline Low & $6(85.7)$ & $37(71.2)$ & $1(14.3)$ & $15(28.8)$ & $0.411(0.045-3.71)$ & 0.661 \\
\hline Moderate and high & $1(9.1)$ & $20(66.7)$ & $10(90.9)$ & $10(33.3)$ & $20.00(2.23-166.6)$ & 0.001 \\
\hline$P$-value for interaction & & & & 0.006 & & \\
\hline
\end{tabular}

MSS/MSI-L, Microsatellite stable / Microsatellite instability low; MSI-H, Microsatellite instability High; *odds of MSS/MSI-L with high BMI within each strata or subgroups

high BMI with MSS/MSI-L was only shown in patients younger than 40 years $(\mathrm{OR}=14.9(1.47-142.9))$ and not for older patients, (OR 1.84 (0.49-6.8), p for interaction was $=0.10$ ). For gender, the association of BMI and MSI was significantly evident among females, as obese were more likely MSS/MSI-L (OR=8.06 (1.44-45.5)) but not among males $(\mathrm{OR}=1.83(0.44-7.63)), \mathrm{p}$ for interaction $=0.187$. When the relation was tested among ever and never smokers, the association of BMI with MSI was only evident among never smokers with a borderline significant interaction, $\mathrm{p}=0.078$. Regarding pathologic characteristic of the tumor, the relationship of BMI with MSI was significant only in stage III but not in early stage (I+II), for those with non-mucin secreting adenocarcinoma with non-significant $\mathrm{p}$ value for interaction $(0.198$ and 0.194 respectively). In patients with low physical activity ( $<600$ MET minutes / week) association of BMI with
MSI was not shown $(\mathrm{OR}=0.41$ (0.04-3.7) but evidence of increased risk of MSS/MSI-L in obese patients was evident in group with moderate to high physical activity $(\mathrm{OR}=20(2.2-166.6), \mathrm{p}$ for interaction $=0.006$ (Table 3$)$

\section{Discussion}

Though CRC is not one of the five most common cancers in Egypt, yet its presentation and prognosis are different from countries where it is one of the three common cancers. Age of incidence is at least one to two decades earlier than that in Western countries (Zeeneldin et al., 2012). This can be due to differences in population structures and in life expectancies. Rockhill and Giovannucci (1999) reported that Westernization of Egypt is occurring and affecting young people, as they are more likely to change their lifestyle compared to older people. 

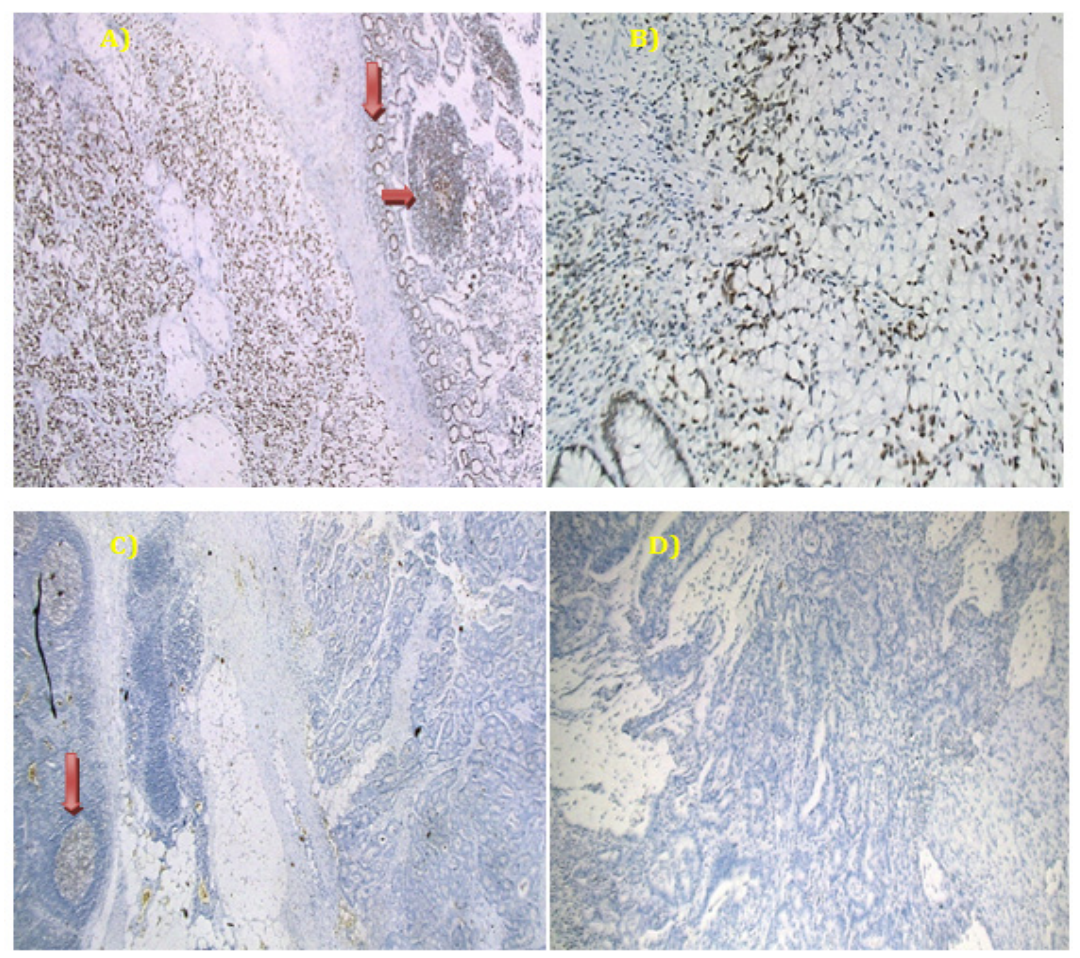

Figure 2. A Representative Example of dMMR (MSI-H) in a Case of Adenocarcinoma with Signet Ring Differentiation. (A) Positive MLH-1 in tumor cells with positive internal control [normal colonic epithelium and lymphoid follicles, red arrows] (Mag. 50X). (B) Positive PMS2 in tumor cells (Mag. 100X). (C) Loss of MSH2 in tumor cells, while positive internal control (lymphoid follicles, red arrow) (Mag. 50X) and (D) loss of MSH6 in tumor cells (Mag 100X).

Because it is a form of heterogeneous cancer, there is interplay of both genetic and environmental factors (Nojadeh et al., 2018). In this study, we aimed at probing the association between life style factors and MSI status in $\mathrm{CRC}$, which could provide more insights on its carcinogenic process.

\section{MSI and relation to patient characteristics and life-style}

Results of the present study showed a higher prevalence of MSI-H group (36.0\%) compared to $15-20 \%$ MSI display in other studies (Vilar and Gruber et al., 2010). An Egyptian study by Nour El Hoda et al., (2017) showed a comparable percentage of MSI-H (30.8\%). Previous study comparing Egyptian CRC patients with Western CRC patients, found that the frequency of MSI-H tumors in the Egyptian group was higher than that in the Western sporadic group (37\% vs. 13\%, p = 0.002) (Soliman et al., 2001). Soliman et al., (2019) found that the expression level of MSI-H was $47.0 \%$, which was more than $10 \%$ higher than the present results. However, many Egyptian studies on MSI prevalence in CRC patients showed that it was higher than that reported from western population, yet there was no clear explanation for this difference. The remarkable differences in molecular pathology as compared to Western patients could result from inherent differences in sensitivity and molecular responses to Western lifestyle in Egyptians (Rockhill and Giovannucci, 1999). Overall, it may be concluded that pathogenesis of $\mathrm{CRC}$ in Egypt has a unique feature, which need extensive investigations.

Regarding patients, demographics age and gender were not significantly related to MSI status. These results were parallel to that by Hoffmeister et al.,(2013) and Kang et al., (2018) who found no relation between MSI and age at diagnosis, but the latter found that proportion of women were significantly higher than men among those with MSI-H CRC than MSS group.

A significant relationship was found between MSI status and tumor site where the left colon and rectum tumors tend to be more MSS while right colon tumors which tend to be more MSI-H. Results by Huang et al., (2010) and Frey et al., (2010) were matched to the current study. Jenkins et al., (2007) and Greenson et al., (2009) found that right location was also a strong predictor of MSI-H. On the contrary, Faghani et al., (2012) found that $81.8 \%$ of total MSI-H had distal tumors. The different methods used by Faghani et al., (2012) to analyze MSI frequencies through using BAT-26 and BAT-25 markers could explain the last finding.

In the present study, high-grade tumors tend to be more MSI-H without significance. Several studies found that poorly differentiated or undifferentiated histology is a strong predictor of MSI-H (Yearsley et al., 2006; Greenson et al., 2009; Frey et al., 2010; Jenkins et al., 2010; Nour El Hoda et al., 2017). On the contrary, Joel et al. (2003) found that the presence of well-differentiated tumors were important markers for microsatellite instability. Huang et al., (2010) found that MSI-H tumors were more likely to show less local aggressiveness and lower differentiation.

A significant association between smoking cigarettes and colon cancer risk have been reported in only few studies, with associations being null or weak and risk estimates of around 1.3-1.4 (Nishihara et al., 2015; Jass et al., 2007; Guastadisegni et al., 2010; Chen et al.,2015). 
Studies that found a statistically significant association between cigarette smoking and colon cancer incidence indicate that dose, or amount usually smoked per day (Jass et al., 2007), is an important predictor of risk. Other studies (Moher et al., 2009; Stroup et al., 2000) that supported the dose response relationship showed stronger associations, with a relative risk of approximately 2.0 , for individuals who reported smoking for 45 years or more. Current study did not find any relationship between smoking as ever and never smoking and MSI status. This could be explained by lower prevalence of ever smoker (29.0\%) and current smokers (11.0\%). Also $100 \%$ of females were never smoker and they constituted $56.0 \%$ of the study group. History of alcohol consumption was not found to be related to MSI as only three patients have history of alcoholic intake. Eight (three cohort and five case-control) studies investigated the association between alcohol intake and CRC according to MSI status. Overall, most studies did not find a significant association between increased alcohol consumption and MSI-H or MSS CRC (Carr et al., 2018).

In this study, neither consumption of diet with high fiber content (vegetable and fruits) nor fast food diet or processed meat were found to be associated with MSI status. A meta-analysis of case-case analyses in three studies revealed no significant difference in the association of dietary fiber intake and MSI-H colon cancer compared with MSS/MSI-L colon cancer (Carr et al., 2018).

We found that patients with low physical activity ( $<600$ MET minutes /week) were more likely to be MSS/MSI-L, $(\mathrm{OR}=2.56 ; 95 \% \mathrm{CI}=1.106$ to 5.925$)$ $\mathrm{p}$ value $=0.026$. Slattery et al., (2000) in the case-control part of study, investigated the association between physical activity and colon cancer by MSI status in both males and females. For males, intermediate and low levels of physical activity were risk factors for both MSI-H and MSS CRC. Among females, low levels of physical activity were associated with an increased risk of MSS/ MSI-L colon cancer but not for MSI-H colon cancer. This relationship was not found in the case-case part of their study. Relation between low physical activity and MSS could be explained by the fact that low physically active persons tend to have higher BMI that increases risk of MSS (Campbell et al., 2007).

High BMI is an established risk factor for colorectal cancer; current study showed that the higher the BMI the more likely MSS/MSI-L tumors, $\mathrm{p}$ value for trend 0.006 . This was supported by Campbell et al., (2007), who found that BMI, per $5 \mathrm{~kg} / \mathrm{m}^{2}$, was positively associated with the risk of MSS $(\mathrm{OR}=1.38 ; 95 \mathrm{CI}=1.24$ to 1.54$)$ and MSL $(\mathrm{OR}=1.33 ; 95 \mathrm{CI}=1.04$ to 1.72$)$ colorectal tumors. Satia et al., (2005) found that high BMI before diagnosis was associated with the risk of MSL or MSS tumors. These results are generally consistent with those of Campbell et al., (2007), providing further evidence for the specific relationship between obesity and the risk of MSS cancer. The specificity of this link not only reinforces a causal interpretation but also points the way to a specific mechanism.

Association of MSI, BMI and interaction with patient characteristics
Interaction mean that two or more factors modifies the effect of each other with regard to an outcome (Szklo and Nieto, 2019). The highly significant relation between BMI $\&$ MSI status was tested for effect modification according to patient characteristics, lifestyle factors and for clinico pathologic prognostic factors.

In this study, we found a strongly elevated risk of MSS/MSI-L CRC associated with high $\left(\geq 25.0 \mathrm{Kg} / \mathrm{m}^{2}\right)$ BMI. This association was limited to younger age $(<40$ years old), females, never smoking, stage III, non-mucin secreting adenocarcinoma and moderate to high physically active patients.

For age, evidence of association of high BMI and MSS/MSI-L was found in young patients ( $<40$ years). Where patients with BMI $(\geq 25.0 \mathrm{Kg} / \mathrm{m} 2)$ and $<40$ years were more susceptible for MSS/MSI-L (OR=14.9 (1.47-142.9), $\mathrm{p}=0.02$ ) while odds for age $\geq 40$ years was $(1.8(0.49-6.8), p=0.488)$ and $p$ for interaction $=0.10$. This result could be explained by Westernization of Egypt affecting younger people, as they are more likely to change their lifestyle compared to older people (Rockhill and Giovannucci, 1999). Parallel with our results, Campbell et al., (2007) found that high BMI at age of 20 years, modeled as a continuous variable in $5 \mathrm{Kg} / \mathrm{m}^{2}$ increment, was positively associated with risk of MSI/L but not MSS or MSI-H. This difference could be explained by difference in MSI definitions in this study as we used two levels of MSI (high versus stable and low), whereas Campbell et al., (2007) characterized three level (MSS, MSL and MSI-H). On the contrary, Hoffmeister et al., (2013), (in the case only part of their study) found that the relation between BMI and MSI was not modified by age categorized as less than 70 and $\geq 70$ years $(\mathrm{OR}=1.37(0.97-1.92), 1.51$ $(1.07-2.12)$ respectively, $p$ for interaction $=0.59$. Difference in results between present study and their study could be explained by different categorization of age.

In the present study, the association of BMI and MSI was shown to be affected by gender, where females odds of being MSS/MSI-L with high BMI was 8.06 (1.44-45.5) compared to those with low BMI, $\mathrm{p}=0.014$. For males this relationship was not evident $(\mathrm{OR}=1.83(0.44-7.63), \mathrm{p}$ for interaction $=0.187$. Despite the fact that we had small number of both males and females with low BMI, which influenced $p$ value for interaction, yet the relation indicates risk evidence for females to have MSS/MSI-L when they are obese. Complex interplay between hormonal factors, fat distribution and tumor biology could be underlying these sex differences to be further elucidated.

Likewise, a Swedish cohort study found higher risk of MSS CRC for women with high BMI but not for males (Brandstedt et al., 2013). Multivariable analysis of the US Health Professionals follow-up study and the Nurses' health study found increasing risk of MSS CRC with increasing BMI (Carr et al., 2018). On the contrary, Hoffmeister et al., (2013) found that per $5 \mathrm{~kg} / \mathrm{m}^{2}$ BMI the risk of MSI-H for females increased by $84.0 \%$ $(\mathrm{OR}=1.84(1.34-2.52))$ with no evidence among males $\mathrm{p}$ for interaction $=0.02$. An explanation for this difference is that a good proportion of CRC cases in their study was not sporadic but hereditary. Another study found that relationship between BMI and MSS/MSI-L was modified 
by gender but was shown only among males not females (Slattery et al., 2000). Campbell et al., (2007) did not find an interaction between BMI, MSI and gender $p$ for interaction $=0.22$.

The association of BMI with MSI was only evident among never smokers with a borderline significant interaction, $p=0.078$. This result could be justified by the fact that more than half of the study group were females who were never smokers, and only $29.0 \%$ of patients were ever smokers.

For pathologic characteristic of the tumor, the relationship of BMI with MSI was significant only in stage III but not in early stage (I+II) and for those with non-mucin secreting adenocarcinoma with non-significant $\mathrm{p}$ value for interaction ( 0.198 and 0.194 respectively). Levi et al., (2011) showed that tumor histology of mucinous cancer is highly correlated with MSI-high tumor and serves as surrogate marker for MSI-high tumors. They reported that the increased risk of MSS associated with increased BMI was evident only for the non-mucinous cancer; they reported that their results were consistent with those of Campbell et al., (2007). Current results also found that non-mucin secreting tumors are risk for MSS/ MSI-L in patients with high BMI which are analogous to Campbell et al., (2007) and Levi et al., (2011) results.

In patients with low physical activity $(<600 \mathrm{MET}$ minutes/ week) association of BMI with MSI was not shown $(\mathrm{OR}=0.41(0.04-3.7))$ but evidence of increased risk of MSS/MSI-L in obese patients was evident in-group with moderate to high physical activity $(\mathrm{OR}=20.0(2.2-166.6)$, $\mathrm{p}$ for interaction $=0.006$. One case-control study (Slattery et al., 2000) investigated the association between physical activity and colon cancer by MSI status and studied the effect of gender on the relation between physical activity and MSI across strata without testing for interaction. In the case control part, they found that for males, intermediate and low levels of physical activity were risk factors for both MSI-H and MSS CRC. Among females, low levels of physical activity were associated with an increased risk of MSS/MSI-L colon cancer but no association was observed for MSI-H colon cancer. In a case-case analysis comparing MSI-H with MSS colon cancer, no difference was identified. Larsson et al., (2006) evaluated the possibility of the interaction between physical activity and $\mathrm{BMI}$ in relation to $\mathrm{CRC}$ risk. They classified participants according to both vigorous leisure-time physical activity and BMI and found that the decreased risk of colorectal cancer associated with increased levels of leisure-time physical activity was observed across all categories of BMI ( $p$ for interaction= 0.33).

In summary, high BMI was strongly associated with MSS/MSI-L CRC, which are known to be bad prognostic subgroups. Though effect modification mostly was not found significant, limited by the relatively small number of subgroups, this relation was getting ready to be influenced by some prognostic factors including, tumor site, TNM stage, tumor pathology and a number of patient's characteristics and life style factors including age, gender and physical activity.

The underlying basis for the increased risk of MSS tumors associated with overweight or obesity and the lack of an association with MSI-H tumors remain largely speculative. However, one possible explanation includes the matrix metalloproteinase system, which is involved in diet-induced obesity through remodeling of the extracellular matrix that surrounds the expanding adipose tissue (Halberg et al., 2008) and in the degradation of the extracellular matrix during colorectal cancer metastasis (Rydlova et al., 2008).

Another potential biological source of increased risk of MS-stable tumors from overweight and obesity not with MSI-H tumors involves telomeres (the physical ends of chromosomes): An inverse association between telomere length and body weight was reported (Kim et al., 2009), and shorter telomere length, in turn, has been linked to chromosomal instability and MS-stable colorectal tumors but not to MSI-high colorectal tumors (Lengauer et al., 1997). Additional studies will be needed to better understand the differing etiologies of MSS/MSI-L and MSI-H colorectal cancers.

Despite the limitations faced, one of the advantages of this study is that, it is the first molecular pathologic epidemiology study probing the effect modification of patient characteristics on the association between BMI and MSI gene in Egyptian patients with extensive data collection. On the other hand, study was limited to 100 patients due to restricted resources and economic difficulties in Egypt, which was reflected on subgroup analyses and multiplicity of comparisons with increased chance of random errors. In addition to a limitation of using IHC that may lead to reaction bias or interpretation bias

In conclusion, this study confirms previous findings of increased risk of MSS/MSI-L colorectal cancer with obesity and suggests that obesity may also be associated with increased risk of MSS/MSI-L colorectal cancer among women, younger patients, late stage, non-mucin secreting adenocarcinoma and physical activity. Further research is needed to confirm present results

\section{Acknowledgments}

\section{Author Contribution}

All authors contributed to the study conception and design. Material preparation, data collection, and analysis were performed by Nora Atef, Nelly Alieldin and Ghada Mohamed. The first draft of the manuscript was written by Nora Atef, Nelly Alieldin and all authors commented on previous versions of the manuscript. All authors read and approved the final manuscript.

\section{Compliance with Ethical Standards}

Funding: the study was funded by Cairo University

\section{Ethical approval}

All procedures performed in studies involving human participants followed the ethical standards of the institutional research committee and with the 1964 Helsinki declaration and its later amendments.

Informed consent

Informed consent was obtained from all individual 
participants included in the study.

\section{Conflict of Interest}

All authors declare that they have no conflict of interest.

\section{Reference}

Arvelo F, Sojo F, Cotte C (2015). Biology of colorectal cancer. Ecancer Med Sci, 9, 520.

Boland CR, Goel A (2010). Microsatellite instability in colorectal cancer. Gastroenterology, 138, 2073-87.

Booth M(2000). Assessment of physical activity: an international perspective. Research quarterly for exercise and sport. Jun 1;71(sup2):114-20.https://doi.org/10.1080/02701367.200 0.11082794

Botteri E, Iodice S, Bagnardi V, Raimondi S, Lowenfels AB, Maisonneuve P (2008). Smoking and colorectal cancer: a meta-analysis. JAMA, 300, 2765-78.

Brandstedt J, Wangefjord S, Borgquist S, et al (2013). Influence of anthropometricfactors on tumour biological characteristics of colorectal cancer inmen and women: a cohort study. J Transl Med, 11, 293.

Campbell PT, Cotterchio M, Dicks E, et al (2007). Excess body weight and colorectal cancer risk in Canada: associations in subgroups of clinically defined familial risk of cancer. Cancer Epidemiol Biomarkers Prev, 16, 1735-44.

Campbell PT, Jacobs ET, Ulrich CM, et al (2010). Case-control study of overweight, obesity, and colorectal cancer risk, overall and by tumor microsatellite instability status. J Natl Cancer Inst, 102, 391-400.

Campbell PT, Newton CC, Dehal AN, et al (2012). Impact of body mass index on survival after colorectal cancer diagnosis: the Cancer Prevention Study-II Nutrition Cohort. $J$ Clin Oncol, 30, 42-52.

Carr PR, Alwers E, Bienert S, et al (2018). Lifestyle factors and risk of sporadic colorectal cancer by microsatellite instability status: a systematic review and meta-analyses. Ann Oncol, 29, 825-84

Chen K, Xia G, Zhang C, et al (2015). Correlation between smoking history and molecular pathways in sporadic colorectal cancer: a meta-analysis. Int J ClinExp Med, 8 , 3241-57.

Faghani M, Asl SF, Ghanaei FM, et al (2012). The correlation between microsatellite instability and the features of sporadic colorectal cancer in the North part of Iran. Gastroenterol Res Pract, 2012, 1-6.

Favoriti P, Carbone G, Greco M, et al (2016). Worldwide burden of colorectal cancer: a review. Updates Surg, 68, 7-11.

Fedirko V, Romieu I, Aleksandrova K, et al(2014). Pre-diagnostic anthropometry and survival after colorectal cancer diagnosis in Western European populations. Int $J$ Cancer, 135, 1949-60.

Frey DM, Droeser RA, Viehl CT, et al (2010). High frequency of tumor-infiltrating FOXP3+ regulatory $\mathrm{T}$ cells predicts improved survival in mismatch repair-proficient colorectal cancer patient. Int $J$ Cancer, 126, 2635-43

Greenson JK, Huang SC, Herron C, et al(2009). Pathologic predictors of microsatellite instability in colorectal cancer. Am J SurgPathol, 33, 126-33.

Guastadisegni C, Colafranceschi M, Ottini L et al (2010). Microsatellite instability as a marker of prognosis and response to therapy: a meta-analysiscolorectal cancer survival data. Eur J Cancer, 46, 2788-98.

Halberg N, Wernstedt-Asterholm I, Scherer PE (2008). The adipocyte as an endocrine cell. Endocrinol Metab Clin North
Am, 37, 753-68.

Harriss DJ, Atkinson G, Batterham A, et al (2009). Lifestyle factors and colorectal cancer risk (2): a systematic review and meta-analysis of associations with leisure-time physical activity. Colorectal Dis, 11, 689-701.

Hoffmeister M, Bläker H, Kloor M, et al (2013). Body mass index and microsatellite instability in colorectal cancer: a population-based study. Cancer Epidemiol Prev Biomarkers, 22, 2303-11.

Huang Y, Yuan Y, Wei-ting GE, et al (2010). Comparative features of colorectal and gastric cancers with microsatellite instability in Chinese patients. Biomed Biotechnol, 11, 647-53.

Hughes LA, Williamson EJ, van Engeland M, et al (2012). Body size and risk for colorectal cancers showing BRAF mutations or microsatellite instability: a pooled analysis. Int $J$ Epidemiol, 41, 1060-72.

Iacopetta B (2002). Are there two sides to colorectal cancer?. Int $J$ Cancer, 101, 403-8.

Jass JR (2007). Classification of colorectal cancer based on correlation of clinical, morphological and molecular features. Histopathology, 50, 113-30.

Jenkins MA, Hayashi S, O'shea A, et al (2007). Pathology features in Bethesda Guidelines predict colorectal cancer microsatellite instability: A population-based study. Gastroenterol, 133, 48-56.

Joel KG, Joseph DB, Ofer BZ, et al (2003). Phenotype of microsatellite unstable colorectal carcinomas: Well-differentiated and focally mucinous tumors and the absence of dirty necrosis correlate with microsatellite instability. Am J Surg Pathol, 27, 563-57.

Kang S, Na Y, Joung SY, et al (2018). The significance of microsatellite instability in colorectal cancer after controlling for clinicopathological factors. Medicine, 97, https://doi. org/10.1097/MD.0000000000010019.

Kawakami H, Zaanan A, Sinicrope FA (2015). Microsatellite instability testing and its role in the management of colorectal cancer. Curr Treat Options Oncol, 16, 30.

Kim S, Parks CG, Deroo LA, et al (2009). Obesity and weight gain in adulthood and telomere length. Cancer Epidemiol Biomarkers Prev, 18, 816-20.

Larsson SC, Rutegård J, Bergkvist L, Wolk A (2006). Physical activity, obesity, and risk of colon and rectal cancer in a cohort of Swedish men. Eur J Cancer, 42, 2590-7.

Lee KJ, Inoue M, Otani T, et al, JPHC Study Group (2007). Physical activity and risk of colorectal cancer in Japanese men and women: the Japan Public Health Center-based prospective study. Cancer Causes Control, 18, 199-209.

Lengauer C, Kinzler KW, Vogelstein B (1997). Genetic instability in colorectal cancers. Nature, 386, 623-7.

Levi Z, Kark JD, Barchana M, et al (2011). Measured body mass index in adolescence and the incidence of colorectal cancer in a cohort of 1.1 million males. Cancer Epidemiol Preve Biomarkers, 20, 2524-31.

Ma Y, Yang Y, Wang F, et al (2013). Obesity and risk of colorectal cancer: a systematic review of prospective studies. PLoS One, 8, e53916.

Moher D, Liberati A, Tetzlaff J, et al (2009). Preferred reporting items for systematic reviews and meta-analyses: the PRISMA statement. BMJ, 339, b2535.

Ning Y, Wang L, Giovannucci EL (2010). A quantitative analysis of body mass index and colorectal cancer: findings from 56 observational studies. Obes Rev, 11, 19-30.

Nishihara R, VanderWeele TJ, Shibuya K, et al (2015). Molecular pathologicalepidemiology gives clues to paradoxical findings. Eur J Epidemiol, 30, 1129-35.

Nojadeh JN, Sharif SB, Sakhinia E (2018). Microsatellite 
instability in colorectal cancer. EXCLI J, 17, 159.

Nour El Hoda SI, El Sheikh SA, Talaat SM, Salem EM (2017). Mismatch repair proteins and microsatellite instability in colorectal carcinoma (MLH1, MSI-H2, MSI-H6 and PMS2): histopathological and immunohistochemical study. Open access Macedonian J Med Sci, $\mathbf{5}, 9$.

Parkin E, O'Reilly DA, Sherlock DJ, Manoharan P, RenehanAG (2014). Excess adiposity and survival in patients with colorectal cancer: a systematic review. Obes Rev, 15, 434-51.

Prizment AE, Flood A, Anderson KE, Folsom AR (2010). Survival of women with colon cancer in relation to precancer anthropometric characteristics: the Iowa Women's Health Study. Cancer Epidemiol Biomarkers Prev, 19, 2229-37.

Raut CP, Pawlik TM, Rodriguez-Bigas MA (2004). Clinicopathologic features in colorectal cancer patients with microsatellite instability. Mutat Res, 568, 275-82.

Renehan AG, Tyson M, Egger M, Heller RF, Zwahlen M (2008). Body-mass index and incidence of cancer: a systematic review and meta-analysis of prospective observational studies. Lancet, 371, 569-78.

Rimm EB, Stampfer MJ, Colditz GA, et al (1990). Validity of self-reported waist and hip circumferences in men and women. Epidemiology, 1, 466-73.

Rockhill B, Giovannucci E (1999). Cancer mortality rates in Menofeia, Egypt: comparison with US mortality rates. Cancer Causes Control, 345-7. https://doi. org/10.1023/A:1008992606786.

Rydlova M, Holubec L Jr, Ludvikova M Jr, et al (2008). Biological activity and clinical implications of the matrix metalloproteinases. Anticancer Res, 28, 1389-97.

Satia JA, Keku T, Galanko JA, et al (2005). Diet, lifestyle, and genomic instability in the North Carolina Colon Cancer study. Cancer Epidemiol Biomarkers Prev, 14, 429-36.

Sinicrope FA, Foster NR, Yoon HH, et al (2012). Association of obesity with DNA mismatch repair status and clinical outcome in patients with stage II or III colon carcinoma participating in NCCTG and NSABP adjuvant chemotherapy trials. J Clin Oncol, 30, 406-12.

Slattery ML, Curtin K, Anderson K, et al (2000). Associations between cigarette smoking, lifestyle factors, and microsatellite instability in colon tumors. $J$ Natl Cancer Inst, 92, 1831-6.

Soliman AS, Bondy ML, El-Badawy SA, et al (2001). Contrasting molecular pathology of colorectal carcinoma in Egyptian and Western patients. Br J Cancer, 85, 1037.

Soliman NA, Morsia DF, Helmy NA(2019). Immunohistochemical Expression of MMR proteins with clinicopathological correlation in colorectal cancer in Egypt. Open Access Macedonian J Med Sci, 7, 1608.

Stroup DF, Berlin JA, Morton SC et al (2000). Meta-analysis of observational studies in epidemiology: a proposal for reporting. Meta-analysis of observational studies in epidemiology (MOOSE) group. JAMA, 283, 2008-12.

Szklo M, Nieto FJ (2019). Epidemiology: beyond the basics. Jones and Bartlett Publishers.

Vilar E, Gruber SB (2010). Microsatellite instability in colorectal cancer e the stable evidence. Nat Rev Clin Oncol, 7, 153-62.

World Cancer Research Fund/American Institute for Cancer Research (2007). Food, nutrition, physical activity, and the prevention of cancer: a global perspective. Washington, DC: AICR.

Yamauchi M, Morikawa T, Kuchiba A, et al (2012). Assessment of colorectal cancer molecular features along bowel subsites challenges the conception of distinct dichotomy of proximal versus distal colorectum. Gut, 61, 847-54.

Yearsley M, Hampel H, Lehman A, et al (2006). Histologic features distinguish microsatellite-high from microsatellite-low and microsatellite-stable colorectal carcinomas, but do not differentiate germline mutations from methylation of the MLH1 promoter. Human pathol, 37, 831-8.

Zeeneldin AA, Saber MM, El-Din IS, Farag SA(2012). Colorectal carcinoma in gharbiah district, Egypt: Comparison between the elderly and non-elderly. $J$ Solid Tumors, 2, 13.

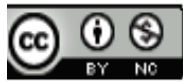

This work is licensed under a Creative Commons AttributionNon Commercial 4.0 International License. 